\title{
Promotion of $\alpha$-Cyano-4-Hydroxycinnamic Acid and Peptide Cocrystallization within Levitated Droplets with Net Charge
}

\author{
Michael J. Bogan, Samuel F. W. Bakhoum, and George R. Agnes \\ Department of Chemistry, Simon Fraser University, Burnaby, British Columbia, Canada
}

\begin{abstract}
Many reactions occur as a result of charge imbalance within or between reactive species in reaction vessels that have zero net charge. Here, chemical processes taking place within reaction vessels having net excess charge were studied. For mass spectroscopists, a familiar example of vessels that defy electroneutrality are the charged droplets produced by an electrospray ion source. Evidence is presented that control of the magnitude of the net charge contained in a reaction vessel, in this case a levitated droplet, can be used to promote nucleation and crystal growth of a mixture of an organic acid, $\alpha$-cyano-4-hydroxycinnamic acid (CHCA), with one or more peptides. This phenomenon was first observed during our ongoing development of wall-less sample preparation (WaSP), electrodynamic charged droplet processing methodology capable of creating micrometer-sized sample spots for matrix-assisted laser desorption/ionization time-of-flight mass spectrometry (MALDI-TOFMS) from subnanoliter volumes of sample material. Peptide ion signal-to-noise $(\mathrm{S} / \mathrm{N})$ ratios obtained by MALDI-TOF-MS from sample spots created from droplets that had high relative magnitude of net charge were consistently greater than those detected from sample spots created from droplets that had lower net charge. To study this unexpected phenomenon further, WaSP methodology was developed to process different mass-to-charge $(\mathrm{m} / \mathrm{z})$ droplets levitated in an electrodynamic balance (EDB), facilitating their deposition onto different positions of a target to create arrays of droplet residues ordered from highest to lowest $\mathrm{m} / \mathrm{z}$. This capability allowed simultaneous levitation with subsequent separation of a population of droplets created from a single starting solution, but the droplets had varied magnitudes of net charge. After the droplets were ejected from the EDB and collected on a glass slide or MALDI plate, the solids contained in the deposited droplets were characterized using microscopy and MALDI-TOF-MS. Factors impacting the chemical processing in droplets having net excess charge levitated in an EDB are discussed with particular emphasis on their possible roles in the promotion of crystal nucleation and growth. (J Am Soc Mass Spectrom 2005, 16, 254-262) (C) 2004 American Society for Mass Spectrometry
\end{abstract}

$\mathrm{T}$ he physics of droplets that possess net charge is a subject that continues to receive considerable theoretical and experimental attention 140 years after initial independent studies by Lord Kelvin and Lord Rayleigh [1-7]. Less well characterized is the condensed phase chemistry that could occur within such droplets as a result of the net charge that is localized in the diffuse layer at the droplet-air interface $[8,9]$. Such chemistry could be rich. For instance, mass spectrometry studies of cluster ions released from charged droplets in an electrospray have identified shifts in chemical equilibria and the formation of preferred nanocrystalline structures [10-14]. It is possible that these perturbations in the chemical processing are dominated by effects that transpire because the reaction vessels defy electroneutrality.

Published online December 21, 2004

Address reprint requests to Dr. G. R. Agnes, Department of Chemistry, Simon Fraser University, Burnaby, B.C., V5A 1S6, Canada. E-mail: gagnes@sfu.ca
Here we apply electrodynamic levitation technology [15-17], microscopy, and MALDI-MS to characterize chemistry that occurred within levitated droplets as a function of the magnitude of their net charge. Droplets were dispensed from a solution containing a MALDI matrix (CHCA) and one or more peptides. Following a brief period of levitation in an EDB, droplets with different $\mathrm{m} / \mathrm{z}$ were sorted by their order of ejection from the EDB and subsequent deposition onto a target. Solids that had formed within the levitated droplet residues were then characterized using microscopy and MALDIMS. It is understood that the key to the detection of intact biomolecules by MALDI-MS is that they are either cocrystallized with a matrix or sorbed onto the surface of matrix crystals so that efficient desorption/ ionization occurs upon laser irradiation [18]. Detection of peptide ions at high $\mathrm{S} / \mathrm{N}$ using lower relative laser irradiation energy from the solids formed in these studies was therefore interpreted as favorable peptide interactions with matrix crystals that had been present. 


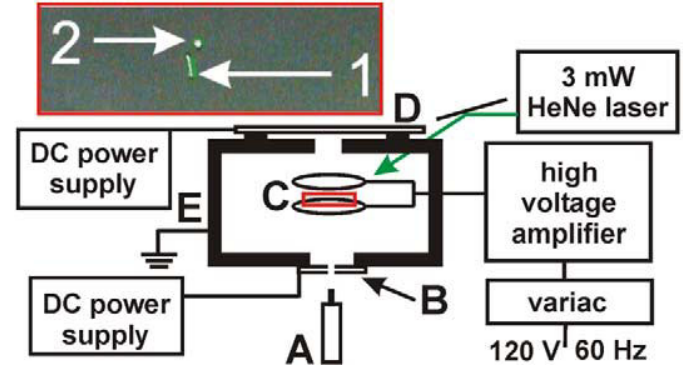

Figure 1. Schematic of the droplet levitation chamber. (a) Droplet dispenser, (b) induction electrode, (c) double-ring EDB, (d) translatable target (MALDI plate or glass slide), (e) levitated chamber fabricated from aluminum and grounded. Inset: (1) $\mathrm{IP}_{\mathrm{f}, 50 \mathrm{~V}}$ and (2) $\mathrm{IP}_{\mathrm{f}, 150 \mathrm{~V}}$ droplets levitated simultaneously.

Evidence is introduced to support the conclusion that during the period of time droplets were levitated, $\mathrm{CHCA} /$ peptide cocrystallization was promoted dependent on the magnitude of the droplet's net charge. This observation has implications for the preparation of MALDI sample spots, and ion and charged cluster generation in an electrospray.

\section{Experimental}

The apparatus for levitation of droplets is depicted in Figure 1. The steps involved in the methodology used to create the droplets and filter them according to their $\mathrm{m} / \mathrm{z}$ are described individually below.

\section{Dispensing of Droplets with Net Charge}

Droplets were created at a rate of $1 \mathrm{~Hz}$ using a piezoelectric droplet-on-demand dispenser (Microfab Technologies Ltd., Plano, TX) fitted with a $40 \mu \mathrm{m}$ diameter orifice (Figure 2). Two starting solutions were used in the cocrystallization studies. A single component peptide solution was prepared by mixing $40 \mu \mathrm{L}$ of acetone, $100 \mu \mathrm{L}$ of a saturated solution of CHCA in 50:50 acetonitrile (ACN): $0.1 \%$ trifluoroacetic acid (TFA) in $\mathrm{H}_{2} \mathrm{O}, 60 \mu \mathrm{L} 20 \%$ glycerol in $\mathrm{H}_{2} \mathrm{O}$, and $50 \mu \mathrm{L}$ of $10 \mu \mathrm{M}$ adrenocorticotropic hormone fragment 18-39 (ACTH) in $\mathrm{H}_{2} \mathrm{O}(0.1 \%$ TFA). A multi-component peptide solution composed of $40 \mathrm{fmol} / \mu \mathrm{L}$ angiotension II and bradykinin, $4 \mathrm{fmol} / \mu \mathrm{L}$ angiotension $\mathrm{I}, 0.2 \mathrm{mg} / \mathrm{ml}$ CHCA, 20\% methanol, $20 \%$ ACN, $1.5 \%$ glycerol, and $0.6 \%$ TFA in $\mathrm{H}_{2} \mathrm{O}$ was also prepared. An aliquot of the starting solutions was loaded into the $\sim 5 \mu \mathrm{L}$ reservoir of the droplet dispenser using a $10.00 \mu \mathrm{L}$ automatic pipette.

A time dependent waveform was applied to the cylindrical piezoceramic element bonded to the outside of the liquid sample reservoir of the droplet dispenser to cause a momentary constriction of the piezoceramic. The resulting pressure wave inside the liquid reservoir forced a jet of liquid from the orifice of the droplet dispenser. An electrode, referred to as the induction electrode, was positioned $3 \mathrm{~mm}$ from the orifice of the droplet dispenser. Variation of the DC potential applied to the induction electrode $\left(\mathrm{IP}_{\mathrm{f}}\right.$, the induction potential during droplet formation) proportionally varied the magnitude of the image charge imparted onto the jet of liquid, such that when the jet of liquid collapsed, a droplet that had net charge resulted. The induction electrode had a $2 \mathrm{~mm}$ diameter hole that had been aligned directly over the top the orifice of the droplet dispenser. Droplets were directed through the hole in the induction electrode and into the center of an EDB.

\section{Measurement of Initial Droplet Net Charge and Volume}

The net excess charge carried by single droplets was measured by dispensing droplets directly onto a stainless steel plate and was found to increase linearly with $\mathrm{IP}_{\mathrm{f}}$ (Figure 2). The plate was connected to the measurement lead of an electrometer (6517a, Keithley Instruments, Cleveland, $\mathrm{OH}$ ), and for these measurements, the droplet dispenser and the plate were housed inside a Faraday cage. In moving this plate from the laboratory bench into a Faraday cage, the background charge fluctuation on the plate as measured by the electrometer decreased by several orders of magnitude to $\pm 5 \mathrm{fC}$. The average charge imparted onto each droplet using a waveform amplitude of $30 \mathrm{~V}$ applied to the droplet dispenser ranged from $37 \mathrm{fC}$ at $\mathrm{IP}_{\mathrm{f}}=50 \mathrm{~V}$, to $437 \mathrm{fC}$ at $\mathrm{IP}_{\mathrm{f}}=250 \mathrm{~V}$.

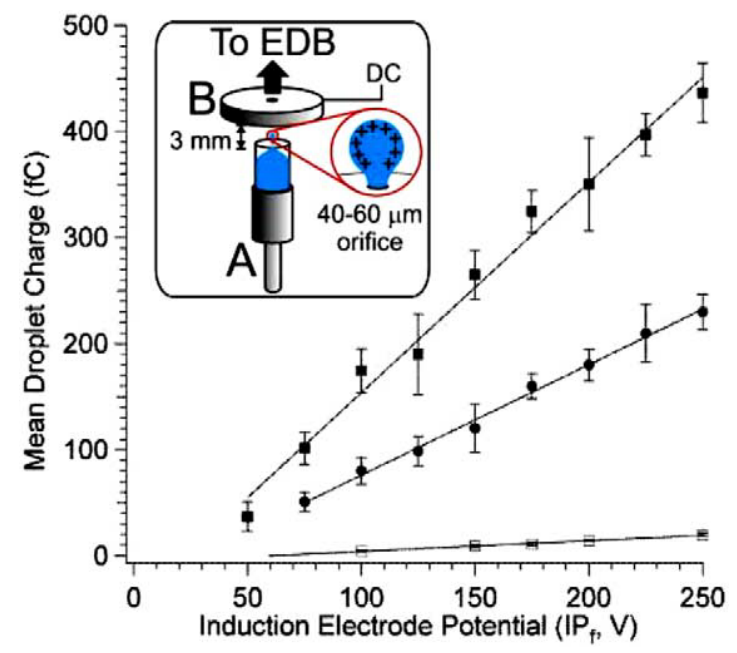

Figure 2. The mean net excess charge carried by a single droplet as a function of the amplitude of the DC potential applied to the induction electrode and the amplitude of the waveform applied to the droplet dispenser. Data points for droplets dispensed with $\pm 10 \mathrm{~V}$ (open squares), $\pm 20 \mathrm{~V}$ (filled circles), and $\pm 30 \mathrm{~V}$ (filled squares) applied to the droplet dispenser piezoceramic were the average of 100 droplets dispensed at $100 \mathrm{~Hz}$ (open symbols) or single droplets at $0.5 \mathrm{~Hz}$ (filled symbols), respectively. The solid lines are linear fits of the data $\left(R^{2}>0.99\right)$. The inset, not to scale, depicts the orientation of the glass-tipped droplet dispenser (a) with respect to the copper induction electrode (b). The polarity of the charge induced on the forming droplet is opposite the polarity of the DC potential applied to the induction electrode. 
The average volume of each droplet dispensed was determined using liquid scintillation. A $100 \mu \mathrm{L}$ stock solution containing 3.7 $\mathrm{MBq}^{32} \mathrm{P}$ labeled orthophosphate in a mixture of $89.2 \%$ water, $10 \%$ methanol, and $0.8 \%$ glycerol was prepared. $5.00 \mu \mathrm{L}$ of this solution was loaded into the droplet dispenser and single droplets were dispensed directly into liquid scintillation vials. To calibrate the radionuclide decay count rate from the vials into which single droplets were dispensed, a 1.000 $\pm 0.005 \mu \mathrm{L}$ aliquot of the ${ }^{32} \mathrm{P}$ in glycerol stock solution was also added into a scintillation vial. $2 \mathrm{~mL}$ of scintillation cocktail (Amersham Biosciences, Baie d'Urfe, Quebec) was added to all of the scintillation vials and then they were vortexed for $30 \mathrm{~s}$. All activity measurements from single droplets and stock solution aliquots were performed using a liquid scintillation counter (LKB Wallac 1217 RackBeta, Fisher Scientific, Montreal, Quebec). Each measurement had an integration time of $10 \mathrm{~min}$. For radionuclide-containing single droplets created when using a voltage amplitude of $30 \mathrm{~V}$ applied to the piezoelectric crystal of the droplet dispenser, the average activity measured was $0.026 \pm 0.001 \%$ that measured from a $1.000 \mu \mathrm{L}$ sample. Thus, the average initial volume of the droplets dispensed was calculated to be $2.6 \times 10^{-4} \pm 1 \times 10^{-5} \mu \mathrm{L}$, or $260 \pm 10 \mathrm{pL}$, corresponding to a radius of $39.6 \pm 0.5 \mu \mathrm{m}$. The net charge imparted onto the droplet did not affect the total volume of solution per droplet dispensed, as determined using radionuclide scintillation counting. Standardized safety protocols for the handling of radionuclides were followed.

Based on these measurements, the droplets used in this work were not expected to undergo Coulomb explosion. The largest net charge carried by droplets used in this study was $\sim 450 \mathrm{fC}$. Following evaporation of the volatile solvents from the droplet, the residues of these droplets, still levitated in the EDB, would have been $12.5 \pm 0.5 \mathrm{pL}$ (droplet radius of $14.4 \pm 0.2 \mu \mathrm{m}$ ). For a droplet containing $450 \mathrm{fC}$, the critical size for Coulomb explosion [2], $\mathrm{R}>\left[(\mathrm{qe})^{2}\left(64 \pi^{2} \varepsilon_{\mathrm{o}} \gamma\right)^{-1}\right]^{1 / 3}$ is $3.85 \mu \mathrm{m}$, where $\mathrm{q}$ is the number of elementary charges per droplet, e the charge of an electron, $\varepsilon_{\mathrm{o}}$ the vacuum permitivity, $\gamma$ the surface tension (assume a 100\% glycerol droplet) and $\mathrm{R}$ the droplet radius. A glycerol droplet of radius $14.4 \mu \mathrm{m}$ can carry a maximum of $2 \times$ $10^{7}$ charges $(1250 \mathrm{fC})$, almost three times the largest net charge induced onto droplets used in this work.

\section{Droplet Levitation}

The electrodynamic levitation technology used here has been described in detail elsewhere [19-21]. An EDB uses DC and AC potentials applied to electrodes to levitate micrometer sized entities that have net charge [22]. Equations that describe ion motion within these devices are known [23]. The ring electrodes of the EDB were fabricated using two copper wires $(0.9 \mathrm{~mm}$ diameter) shaped into $2 \mathrm{~cm}$ diameter rings and mounted parallel at a separation distance of $6 \mathrm{~mm}$. A sine wave ranging in amplitude from 500 to $2,700 \mathrm{~V}_{0-\mathrm{P}}$ at $60 \mathrm{~Hz}$ was applied to the ring electrodes $\left(A C_{\text {trap }}\right)$, in phase. The induction electrode and a flat plate, referred to as the target, served as the endcap electrodes of the EDB. DC potentials were applied to these two electrodes to manipulate the vertical location of the levitated droplets. A $4 \mathrm{~mW}$ green HeNe laser (Uniphase model 1676, Manteca, CA) was used to illuminate the droplets levitated inside the EDB, and images of them were collected by focusing a digital camera through a microscope objective to the center of the ring electrodes.

A noteworthy characteristic of an EDB is that the time scale of the levitation can be set to vary from milliseconds to days, provided the droplet's $m / z$ remains within the EDB's region of stability. Either static or programmed changes in the potentials applied to the electrodes comprising the EDB could be used to cause the droplet to be ejected when its $m / z$ reaches a certain critical value, or those potentials can be set to retain the droplet within the EDB indefinitely. Importantly, the ejection of each droplet from the EDB can be controlled, and each droplet can be deposited at a known location on a substrate which allows subsequent manipulation and characterization of it [19]. Furthermore, translation of the substrate plate between droplet deposition events allows arrays of droplet residues to be formed [19], and it will be shown that they are ordered according to the $m / z$ of the levitated droplet residues.

\section{Matrix-Assisted Laser Desorption/Ionization Mass Spectrometry}

MALDI-MS spectra of the components in individual or codeposited droplet residues, referred to as sample spots on the substrate, were acquired using two different TOF-MS instruments, a Voyager-DE (Perseptive Biosystems, Framingham, MA) and a MALDI-LR (Waters Technologies Inc., Manchester, UK). In all cases, the output of the focused laser was positioned overtop of each spot prior to firing the laser [20].

The energy of laser irradiation reaching the sample was able to be adjusted in one of three coarse steps by placing one of two neutral density filters that had different optical density, or no filter in the path of the laser output. Fine control of the laser irradiation energy was provided by a variable size aperture that was also under computer control, and that enabled ready optimization of the $\mathrm{S} / \mathrm{N}$ for one or more analyte compounds.

Each MALDI-MS spectrum is the sum of all ions detected from the indicated number of laser shots fired. Note that glycerol, which was present in all starting solutions to facilitate droplet levitation, is considered detrimental to MALDI-MS analysis. The investigation of preparing MALDI sample spots by WaSP without glycerol is ongoing [21]. 


\section{Results}

First Observations of the Promotion of MALDI

Matrix/Peptide Cocrystallization in Levitated Droplet Residues

The motivation for the experiments reported here arose from an unexpected observation made during the ongoing development of methodology, termed wall-less sample preparation (WaSP), for the preparation of micrometer-sized MALDI sample spots from picolitervolume droplets levitated in an EDB. To process droplets using WaSP, they must carry net charge because the apparatus for this methodology uses electric fields to manipulate their trajectories. While investigating the effect of droplet net charge on the peptide ion abundance measured from WaSP-prepared MALDI sample spots, peptide ion signals were repeatedly detected at higher $\mathrm{S} / \mathrm{N}$ from sample spots created from droplets that had higher relative net charge. In these experiments, all droplets were created from starting solutions whose solvent composition contained a large percentage of volatile solvent. Within seconds of the instant of each droplet dispensing event, the volatile solvents evaporated from the levitated droplet leaving behind a droplet residue consisting of any low-volatility solvents (glycerol), and solutes [24]. During that period of time, the droplet's mass decreased by one to three orders of magnitude depending on the starting solution composition and the environmental conditions in the levitation chamber. Optical microscopy of droplet residues deposited on glass slides revealed that droplets with high net charge consistently yielded more MALDI matrix/peptide precipitates than droplets with lower relative net charge, accounting for the higher peptide ion yields observed by MALDI-MS. Had the effects due to rapid solvent evaporation, such as droplet temperature decrease and solute supersaturation alone been responsible for these effects, there would not have been such dramatic differences in the quantity of precipitates in the droplet residues as a function of the magnitude of their net charge.

An example of these results is presented in Figure 3. The single component peptide starting solution was used to generate 10 droplets that were each levitated for 2 min prior to depositing all of them at a single location on a glass slide to create a single sample spot. Optical microscopy of the sample spot composed of 10 droplet residues showed that CHCA precipitates were created during levitation. Insets a-d of Figure 3 show four examples of sample spots formed from a population of 10 droplets created with the induction electrode potential set at $90 \mathrm{~V}$ (referred to hereafter as $\mathrm{IP}_{\mathrm{f}, 90 \mathrm{~V}}$ droplets). MALDI-MS spectra were acquired at a fixed laser irradiation setting from sample spots prepared by codepositing the residues of $10 \mathrm{IP}_{\mathrm{f}, 90 \mathrm{v}}$ droplets onto a MALDI target plate, yet no ion signals were detected that could be attributed to ACTH or a fragment thereof (Figure 3). The experiment was performed again with

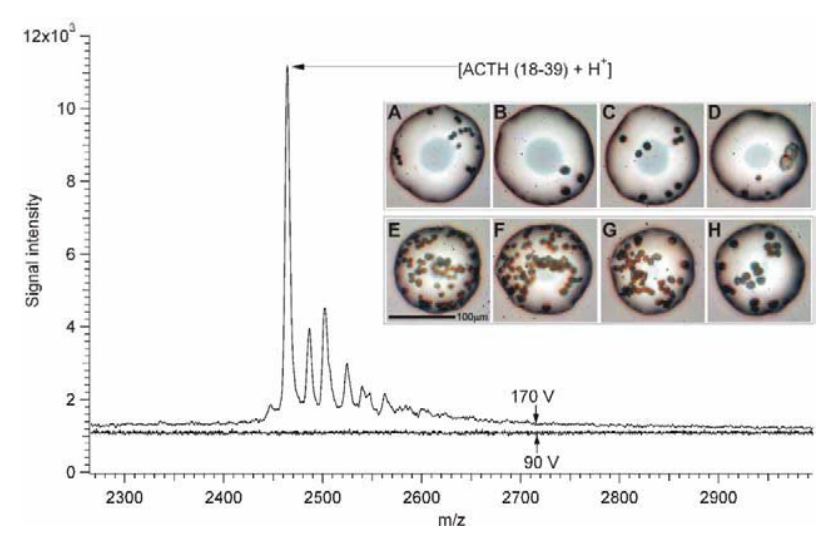

Figure 3. The ACTH ion signal intensity detected from 32 laser shots directed at two discrete sample spots prepared from 10 $\mathrm{IP}_{\mathrm{f}, 170 \mathrm{~V}}$ and $10 \mathrm{IP}_{\mathrm{f}, 90 \mathrm{~V}}$ droplets, respectively. To collect each mass spectrum the laser was directed at the sample spot and held stationary while it was pulsed because all ten droplets that formed each sample spot fit within the laser spot diameter. The data constituting each mass spectrum were subjected to a boxcar average of five points for smoothing purposes and no background subtraction or X-Y offset was performed. Inset: Images of single glycerol droplet residues created from $10 \mathrm{IP}_{\mathrm{f}, 90 \mathrm{~V}}(\mathbf{a}-\mathbf{d})$ or $\mathrm{IP}_{\mathrm{f}, 170 \mathrm{~V}}$ droplets (e-h), levitated in the EDB for 2 min and deposited onto a glass slide. The solids (ACTH cocrystallized with CHCA) inside the deposited residues were formed while the droplets were levitated.

$\mathrm{IP}_{\mathrm{f}, 170 \mathrm{~V}}$ droplets. These sample spots contained substantially more CHCA precipitates (Figure 3 , inset e-h) than the residues of the $\mathrm{IP}_{\mathrm{f}, 90 \mathrm{~V}}$ droplets (Figure 3, inset a-d). Ion signals for the intact peptide ACTH $\left[\mathrm{ACTH}+\mathrm{H}^{+}\right.$, $m / z=2465.2$, were detected by MALDI-MS analysis of sample spots created from the residues of 10 codeposited $\mathrm{IP}_{\mathrm{f}, 170 \mathrm{~V}}$ droplets (Figure 3). The ion signals detected in the region of $m / z=2480-2730$ were ACTH containing clusters with cations $\mathrm{Na}^{+}, \mathrm{K}^{+}$, and solvent molecules. The same laser irradiation energy was used throughout in acquiring the spectra plotted in Figure 3. Precipitate abundance as observed by optical micropscopy, and ion count as detected by MALDI-TOF-MS were similar in sample spots created from levitated droplets with the polarity of the net charge reversed.

The data presented in Figure 3 indicated that the abundance of precipitates formed during the period of time the residues remained levitated in the EDB was dependent on the magnitude of the net charge imparted onto the original droplets. High ACTH ion abundance from the $\mathrm{IP}_{\mathrm{f}, 170 \mathrm{~V}}$ droplet residues reflected the increased amount of ACTH cocrystallized with CHCA relative to the precipitates in the $\mathrm{IP}_{\mathrm{f}, 90 \mathrm{~V}}$ droplet residues. These results were not expected, but were consistent over a series of 20 experiments by two investigators using different solutions, droplet dispensers, and experimental apparatus. Although the increased ion abundance can be explained by the increased MALDI matrix/ peptide crystals observed in the residues of droplets with high net charge, the question of why this differential chemical processing was occurring was not answered. Experimental methodology designed to delin- 
eate the source of this unexpected phenomenon and to better characterize the properties of the initial dispensed droplets, their residues that were levitated in the EDB, and the precipitates that formed in those residues while levitated are described in the remainder of the manuscript.

\section{Levitation and Ejection of Different $\mathrm{m} / \mathrm{z}$ Droplets}

Inducing varied net charge on dispensed droplets of constant volume changed their respective $m / z$. To effectively study droplets of differing $m / z$ using the EDB, it was important to delineate whether droplets of different $m / z$ could be differentiated from each other while levitated in the EDB. First, an individual $\mathrm{IP}_{\mathrm{f}, 50 \mathrm{~V}}$ droplet was levitated. After noting its trajectory, it was ejected and then a single $\mathrm{IP}_{\mathrm{f}, 150 \mathrm{~V}}$ droplet was levitated at the same $\mathrm{AC}_{\text {trap. }}$. Different trajectories for these two droplets were discernible to the naked eye. Next, an $\mathrm{IP}_{\mathrm{f}, 50 \mathrm{~V}}$ and an $\mathrm{IP}_{\mathrm{f}, 150 \mathrm{~V}}$ droplet were levitated simultaneously in the EDB. Again, their trajectories were clearly different (Figure 1 inset). This observation enabled repeat experiments to be performed on two different $m / z$ droplets simultaneously levitated in the EDB because it was possible to visually track which droplet was which.

Another way to differentiate between the two droplets of different $\mathrm{m} / \mathrm{z}$ was developed when the DC potential applied to the target required to eject each droplet was measured. For all repetitions of the experiment, the $\mathrm{IP}_{\mathrm{f}, 50 \mathrm{~V}}$ droplet was ejected from the EDB first and the $\mathrm{IP}_{\mathrm{f}, 150 \mathrm{~V}}$ droplet remained levitated. This observation was consistent with all combinations of two different $m / z$ droplets levitated in the EDB (i.e., $\mathrm{IP}_{\mathrm{f}, 50 \mathrm{~V}}$ droplet ejected before $\left.\mathrm{IP}_{\mathrm{f}, 150 \mathrm{~V}}\right)$. With only two droplets that had the same $m / z$ levitated, such as two $\mathrm{IP}_{\mathrm{f}, 50 \mathrm{~V}}$, $\mathrm{IP}_{\mathrm{f}, 100 \mathrm{~V}}$, or $\mathrm{IP}_{\mathrm{f}, 150 \mathrm{~V}}$ droplets, the trajectories of the droplets were similar and their deposition potential (DP) values were identical within experimental error [21]. Thus, two separate and complementary procedures were developed to differentiate between two different $\mathrm{m} / \mathrm{z}$ droplets levitated in the EDB, facilitating further experiments designed to characterize differential chemical processing as a function of droplet net charge.

Next, the complexity of the experiment was increased. A population of five droplets was injected into the EDB where each had been created at $\mathrm{IP}_{\mathrm{f}}=100,125$, 150,175 , and $200 \mathrm{~V}$, respectively. The DC potential applied to the induction electrode was adjusted to $0 \mathrm{~V}$ after 2 min of levitation time. The voltage applied to the target was then manually ramped to higher values and DP was measured for each of the five levitated droplets. This procedure was repeated for nine more sets of five droplets. A plot of mean DP versus $\mathrm{IP}_{\mathrm{f}}$ was linear over the range $\mathrm{IP}_{\mathrm{f}}=100$ to $200 \mathrm{~V}$, indicating that none of these droplets underwent a Coulomb explosion (Figure 4a). The mass change between the initial droplet and its levitated residue was therefore able to be determined simply based on evaporation of the volatile solvents contained in the starting solution only.
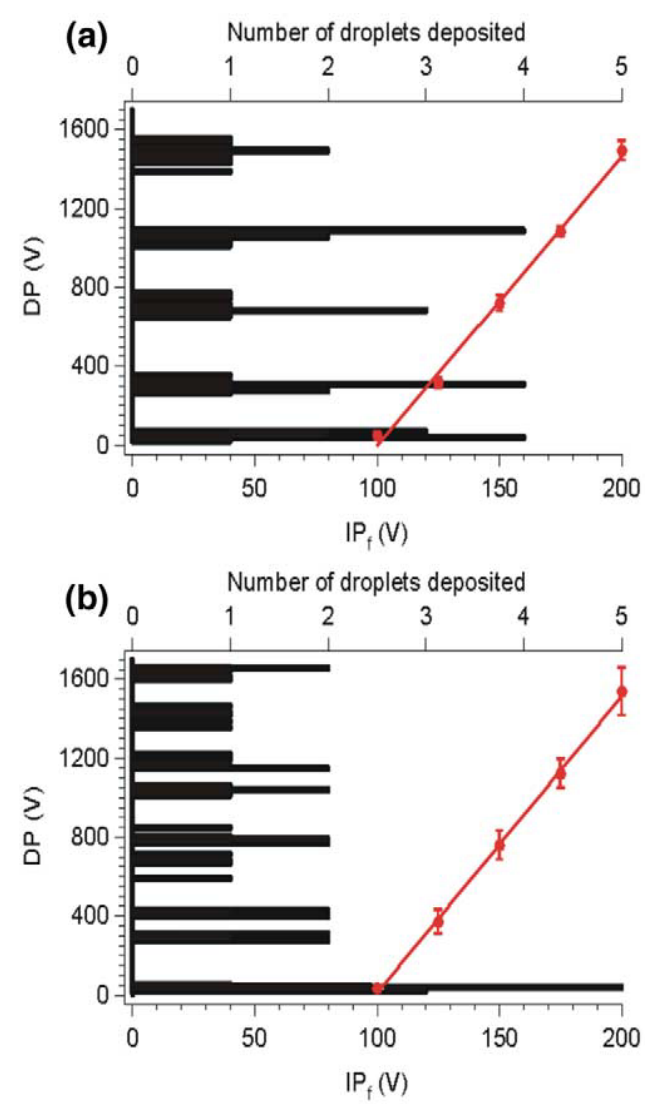

Figure 4. Filtering of levitated droplets as a function of their $\mathrm{m} / \mathrm{z}$ by ejecting them one at a time from an EDB. The mean deposition potential (DP) measured for droplets created at different induction potentials $\left(\mathrm{IP}_{\mathrm{f}}\right)$ and levitated simultaneously for a population of (a) 5 droplets (10 replicates) and (b) 10 droplets (5 replicates, population consisted of 2 droplets at each $\mathrm{IP}_{\mathrm{f}}$ ). Presentation of this data as a histogram of the number of droplets deposited at $15 \mathrm{~V}$ intervals created a data set that could be construed as a mass spectrum of the levitated charged droplets (left axis).

The histogram shown on the left hand axis in Figure 4a displays the number of droplets deposited versus the deposition potential. To build the histogram an arbitrary bin size of $\Delta 15 \mathrm{~V}$ was used to count droplets that were deposited from the EDB. This presentation format of the data shows five clusters corresponding to the droplets that were created at each of the five $\mathrm{IP}_{\mathrm{f}}$ values used, and therefore each cluster represents a collection of droplets of unique $m / z$. The droplets created with $\mathrm{IP}_{\mathrm{f}}=100,125,150,175,200 \mathrm{~V}$ have values of $\mathrm{m} / \mathrm{z}=2.2$ $\times 10^{10}, 1.7 \times 10^{9}, 1.3 \times 10^{9}, 1.1 \times 10^{9}$, and $9.5 \times 10^{8}$, respectively, at the time of ejection from the EDB. The histogram of different $\mathrm{m} / \mathrm{z}$ droplets can be considered analogous to the time-based histogram of ion arrival at the detector of a TOF-MS in specified time periods that is then converted to a $m / z$ axis by calibration using ions of known $m / z$. Therefore, the histogram represents the raw data for $m / z$ filtering of levitated droplets, which can be construed as a mass spectrum. The resolution for ejection of levitated droplets from the EDB was evaluated as 6 using $\mathbf{R}=\left[m / z_{150} /\left(m / z_{150}-m / z_{175}\right)\right]$ where 
(a)

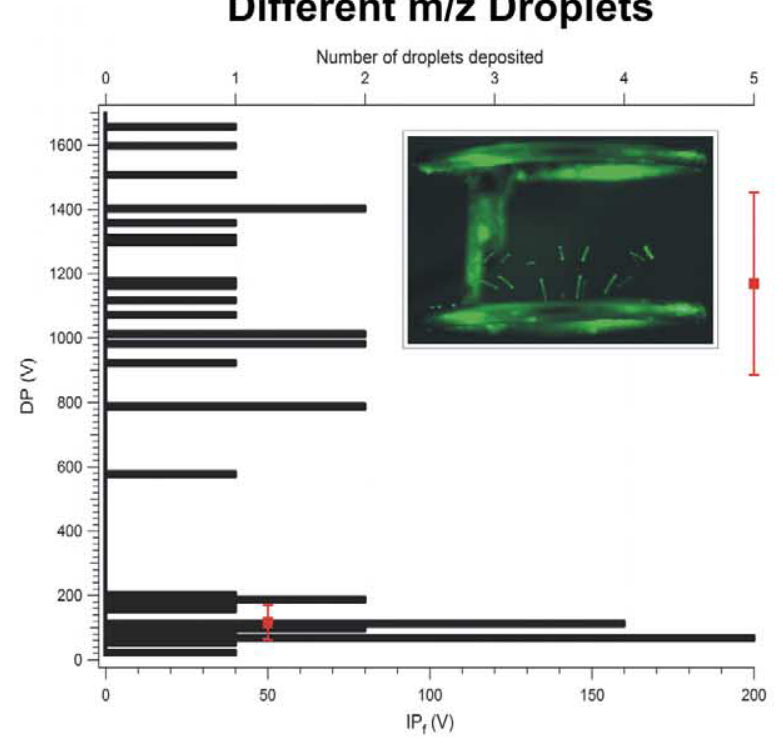

(b) Microscopy of Deposited Droplets
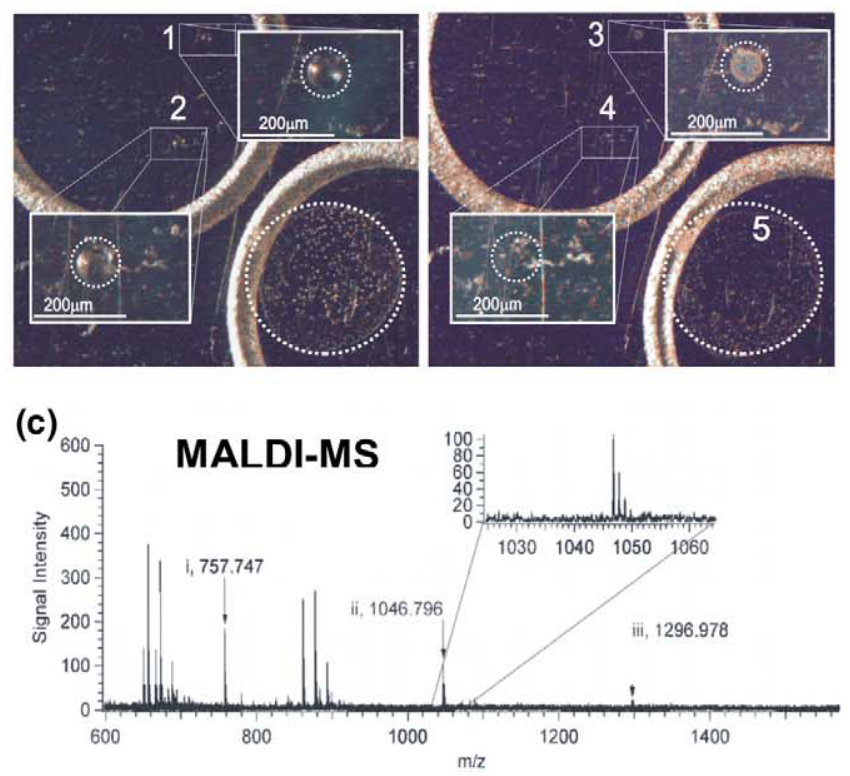

Figure 5. Measurement of differential chemical processing that occurred within droplets whose net charge was 140 or $290 \mathrm{fC}$. (a) Histogram of the number of droplets deposited at $15 \mathrm{~V}$ DP intervals for a population of 40 droplets, $20 \mathrm{IP}_{\mathrm{f}, 50 \mathrm{~V}}$ and $20 \mathrm{IP}_{\mathrm{f}, 200 \mathrm{~V}}$. Inset: Image of droplet population levitated in EDB prior to ejection. (b) Light microscopy of the codeposited droplets before $\left(1=20 \mathrm{IP}_{\mathrm{f}, 200 \mathrm{~V}}\right.$ droplets, $\left.2=20 \mathrm{IP}_{\mathrm{f}, 50 \mathrm{~V}}\right)$ and the same spots respectively after vacuum drying $(3,4)$. The bottom right of the images show a sample spot created from $0.25 \mu \mathrm{L}$ delivered by pipette (5). Sample spot regions are outlined by dotted white circles that are intended to be guides for the eye. (c) MALDI-MS characterization of the solids formed in the residue labeled as 3 . The measured peptide monoisotopic masses are labeled, and the quantity of the following peptides per spot were $\mathrm{i}=$ bradykinin (220 $\mathrm{amol})$, ii = angiotension II $(220 \mathrm{amol})$, and iii = angiotension I $(22 \mathrm{amol})$. No peptide ion signals were detected from sample spot 4 .

$m / z_{150}$ and $m / z_{175}$ represent the $m / z$ values of the $\mathrm{IP}_{\mathrm{f}, 150 \mathrm{~V}}$ and $\mathrm{IP}_{\mathrm{f}, 175 \mathrm{~V}}$ droplets, respectively.

Next, the number of droplets in the EDB was increased to 10 by injecting two droplets created at each distinct $\mathrm{IP}_{\mathrm{f}}$ (Figure $4 \mathrm{~b}$ ). Droplets with $\mathrm{m} / \mathrm{z}$ differences in excess of the resolution for ejection from the EDB were trapped simultaneously and deposited onto the substrate according to their $m / z$, in order from highest to lowest $\mathrm{m} / \mathrm{z}$. However, there were two distinct potentials for the deposition of the first versus the second droplet that had been created using identical conditions. The explanation for this effect is Coulomb repulsion between the two droplets that had $m / z$ values that were within the experimental error of being the same, and as a result, both droplets adopted similar trajectories within the EDB. The result of their repulsive interaction is that the $\mathrm{m} / \mathrm{z}$ resolution for droplet ejection degraded [15]. For comparison, Coulomb repulsion effects impact the resolution in a three-dimensional quadrupole ion trap mass spectrometry when there are $>1 \times 10^{6}$ ions trapped with helium bath gas at a total pressure of 1 mTorr [25-27], so Coulomb repulsion in our experiments was not unexpected because each of the levitated droplets carried a net charge of $\sim 1 \times 10^{6}$ ions.

\section{Methodology for the Study of Chemistry within Droplets with Varied Net Charge}

Using an aliquot of the multi-component peptide starting solution to load the droplet generator, 20 $\mathrm{IP}_{\mathrm{f}, 50 \mathrm{~V}}$ droplets and $20 \mathrm{IP}_{\mathrm{f}, 200 \mathrm{~V}}$ droplets were created (and injected) sequentially into the EDB and those two populations levitated simultaneously (Figure 5a, inset). After the last droplet was injected into the EDB, the potential of the induction electrode was reduced to $0 \mathrm{~V}$. Following a levitation period of $2 \mathrm{~min}$ the 40 droplets were filtered according to their $\mathrm{m} / \mathrm{z}$ upon their ejection from the EDB (Figure 5a). Two spots of material were created during the deposition of droplets from the EDB. One spot was comprised of the first twenty droplets ejected from the EDB $\left(\mathrm{IP}_{\mathrm{f}, 50 \mathrm{~V}}\right.$ droplets), and the second spot was formed by depositing the remaining twenty levitated droplets $\left(\mathrm{IP}_{\mathrm{f}, 200 \mathrm{~V}}\right.$ droplets) onto a different location of the target. These two sample spots are identified as region 1 and 2 in the inset of Figure 5b, and were comprised of the droplets of low $\left(\mathrm{IP}_{\mathrm{f}, 200 \mathrm{~V}}\right)$ and high $\left(\mathrm{IP}_{\mathrm{f}, 50 \mathrm{~V}}\right) \mathrm{m} / \mathrm{z}$, respectively. Note that because the rate of droplet generation was $1 \mathrm{~Hz}$, the $\mathrm{IP}_{\mathrm{f}, 50 \mathrm{~V}}$ droplets were levitated longer than the $\mathrm{IP}_{\mathrm{f}, 200 \mathrm{~V}}$ droplets. This difference in time to form precipitates was not a limit- 
ing factor because there were consistently more precipitates in the $\mathrm{IP}_{\mathrm{f}, 200 \mathrm{~V}}$ droplets that were levitated for a shorter period of time. Attempts to perform this experiment in reverse order of droplet injection were unsuccessful because with $\mathrm{IP}_{\mathrm{f}, 200 \mathrm{v}}$ droplets levitated, the momentum imparted onto the $\mathrm{IP}_{\mathrm{f}, 50 \mathrm{v}}$ droplets during their formation, which facilitated their injection into the EDB, resulted in their passing through the EDB because of Coulomb repulsion from the $\mathrm{IP}_{\mathrm{f}, 200 \mathrm{~V}}$ droplets.

By inserting the MALDI plate into a vacuum chamber $\left(\sim 3 \times 10^{-7}\right.$ torr), the glycerol from these spots evaporated, facilitating observation of the solids that had formed in the levitated droplets by light microscopy (inset 3 and 4, Figure 5b). These two spots of sample were targeted with the $\mathrm{N}_{2}$ laser set at an irradiation threshold of $76 \%$ (75 $\mu \mathrm{J} /$ pulse). Signals corresponding to the three peptides (i: bradykinin, ii: angiotension II, iii: angiotension I) contained in the solution used to make the droplets were detected from the spot comprised of $\mathrm{IP}_{\mathrm{f}, 200 \mathrm{~V}}$ droplets (net charge $=290 \mathrm{fC}$ ) (Figure 5c), but not from the $\mathrm{IP}_{\mathrm{f}, 50 \mathrm{~V}}$ droplets (net charge $=140 \mathrm{fC}$ ). The signal-to-noise ratio $(\mathrm{S} / \mathrm{N})$ of the ions detected from the $220 \mathrm{amol}$ of angiotension II in the $81.5 \mu \mathrm{m}$ diameter sample spot was 11 and only $0.0054 \mu \mathrm{l}$ of sample was consumed. For comparison, a sample spot created by pipetting $0.25 \mu \mathrm{L}$ of the multicomponent peptide starting solution (Figure $5 \mathrm{~b}$, region 5 , $10 \mathrm{fmol}$ of angiotension II in the aliquot) which resulted in a spot diameter of $1390 \mu \mathrm{m}$ diameter, yielded a $\mathrm{S} / \mathrm{N}$ of 37 for angiotension II. Because the mass spectrum from the $0.25 \mu \mathrm{L}$ sample spot was collected without changing the position of the laser spot (200 $\mu \mathrm{m}$ diameter), only $\sim 830$ amol of angiotension II was irradiated. This accounts for the four times decrease in $\mathrm{S} / \mathrm{N}$ observed from the 20 droplet sample spot and therefore essentially identical signals were obtained from that sample spot while consuming 50 times less sample solution.

Another replicate of the experiment was performed to prepare fresh sample spots. A sample spot was created from 12 codeposited $\mathrm{IP}_{\mathrm{f}, 90 \mathrm{~V}}$ droplets and another sample spot was created from 12 codeposited $\mathrm{IP}_{\mathrm{f}, 170 \mathrm{~V}}$ droplets. Each of these spots contained $6 \mathrm{fmol}$ ACTH. The laser output was directed at each of the sample spots and the laser was fired 50 times at the lowest irradiation setting. The ions detected from this set of laser shots were accumulated as five mass spectra (ten laser shots each), and from that, the mean peak height of the monoisotopic ion, $\left[\mathrm{ACTH}+\mathrm{H}^{+}\right]$at $\mathrm{m} / \mathrm{z}=2465.2$ was calculated and plotted with an error of one standard deviation of the mean. The laser irradiation setting was incremented and the analysis repeated (Figure 6a). This set of data shows the peptide ACTH was in fact present in all samples, but its detection was dependent on the laser irradiation energy, which was interpreted as being indicative of the relative quantity of $\mathrm{ACTH}$ in these two samples that had cocrystallized and/or sorbed onto CHCA crystals [28]. The sample with a larger abundance of CHCA crystals offered a greater crystal volume
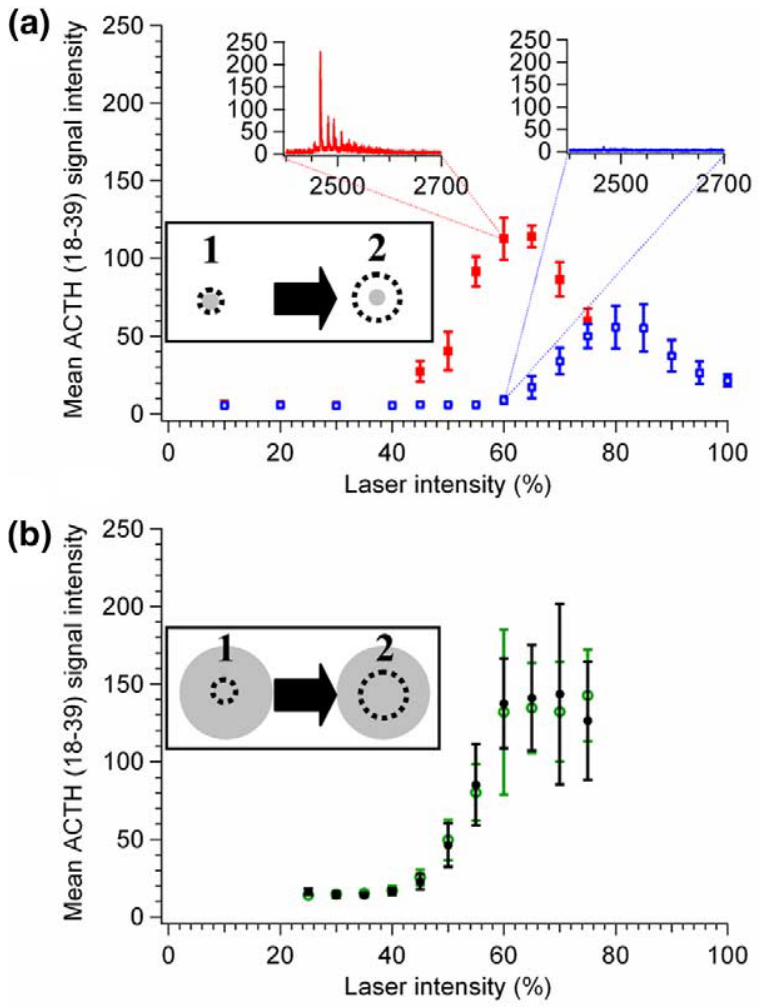

Figure 6. The mean monoisotopic $\left[\mathrm{ACTH}+\mathrm{H}^{+}\right]$peak height from 50 laser shots fired at sample spots created from (a) $12 \mathrm{IP}_{\mathrm{f}, 90 \mathrm{v}}$ droplets $(3 \mathrm{~nL})$ (open squares) or $12 \mathrm{IP}_{\mathrm{f}, 170 \mathrm{~V}}$ droplets (filled squares) and (b) $1.00 \mu \mathrm{L}$ deposited by pipette on a stainless steel MALDI plate that was grounded (open circles) or had +500 V DC applied to it (filled circles). Ion abundances were measured at increments of the neutral density filter that determines the laser irradiation. At the 20 and $100 \%$ settings for laser irradiation energy, the energy per pulse was measured to be 30 and $121 \mu \mathrm{J}$, respectively.

and/or a larger surface area from which peptide compounds could be desorbed, and ion signals for [ACTH $+\mathrm{H}^{+}$] at higher $\mathrm{S} / \mathrm{N}$ were detected at a lower threshold for laser irradiation.

The importance of net charge in the medium during nucleation versus placing an electrode in a medium to raise its potential above ground was investigated. Aliquots of the single component peptide starting solution $(1.00 \mu \mathrm{L}$ aliquot, $319 \mathrm{fmol} / \mu \mathrm{L}$ of ACTH deposited) were pipetted directly onto a MALDI plate and allowed to dry while the MALDI plate was either grounded or biased with a DC potential. Note that in these experiments each aliquot was overall net neutral, and the aliquot spread over a region circular in shape and had a diameter of $1.5 \mathrm{~mm}$ once dry. Within the area irradiated by the $\mathrm{N}_{2}$ laser that was focused to a spot size of $200 \mu \mathrm{m}$ in diameter, there would have been approximately $6 \mathrm{fmol}$ of material. When the $\left[\mathrm{ACTH}+\mathrm{H}^{+}\right]$signal intensity was measured from these sample spots that were large relative to the laser spot size, the signal intensity increased and then stabilized at a plateau (Figure $6 \mathrm{~b})$ as the area of sample irradiated increased as the 
laser fluence increased. This was because fresh sample material was accessed as the laser spot size increased with larger aperture sizes (plotted as percentage laser irradiation setting, x-axis, Figure $6 \mathrm{~b}$, inset). If the laser fluence was increased further, the $\left[\mathrm{ACTH}+\mathrm{H}^{+}\right.$] signal intensity would eventually decrease because no new cocrystallized MALDI matrix/peptide material would be irradiated and that being irradiated was being consumed. The lack of a separation between laser threshold detection of ACTH in the MALDI-MS of these samples suggests that there was no detectable difference in the two preparations with respect to the CHCA crystal abundance or surface area.

\section{Discussion}

The abundance of solids formed in levitated droplets of radius $\sim 15 \mu \mathrm{m}$ each dispensed from the same starting solution was consistently found to be dependent on the magnitude of the droplet's net charge. These results were obtained using identical volume aliquots from the same starting solutions. Visual inspection and MALDI-MS characterization of the levitated droplet residues revealed a substantial increase in the abundance of CHCA solids in droplet residues that had $290 \mathrm{fC}$ of net charge relative to the residues that had $140 \mathrm{fC}$. Both sets of droplets had been created from starting solutions that were well below the CHCA solubility limit. Even after the droplets had been levitated for $\sim 2$ min with most of the volatile solvent removed by evaporation, the mass ratio of CHCA to glycerol that remained was estimated to be $>1: 100$, over an order of magnitude higher than the solubility limit mass ratio of 1:8 [29]. This suggests that the supersaturation concentration of CHCA, necessary for it to form nuclei of the critical size required for spontaneous growth, was dependent on the medium's net charge. When the polarity of the net charge was reversed, similar results were obtained suggesting that the magnitude of the net charge was most significant. Repetition of these experiments with the multi-component peptide starting solution yielded identical results, indicating this phenomenon was not dependent on the nature of the peptide being cocrystallized or sorbed onto CHCA crystals. While it is acknowledged that these observations were made with a single organic acid, CHCA is a simple aromatic organic acid with no unique properties. CHCA was the subject of this work simply because of its prominent use as a MALDI matrix. In consideration of these facts, we have interpreted the results presented herein to mean that the magnitude of the net excess charge carried by the reaction vessel (a levitated droplet) can be used to control the abundance of CHCA crystals formed within it. However, the actual mechanism by which variation in a droplet's net charge caused nucleation of CHCA to be promoted has yet to be elucidated. Possible mechanisms are discussed below.

If the reaction vessel environment is the factor re- sponsible for promotion of crystal growth, which of its specific properties are the cause, and what implications does this hold for crystal nucleation and growth in general? The net charge carried by liquid droplets is a population of ions that are unpaired and they reside in the diffuse layer in droplet-air interface [8]. The net charge carried by the droplets is due to the electrophoretic mobility of ions in the solution during droplet formation and thus the composition of the droplets were modified as a function of $\mathrm{IP}_{\mathrm{f}}$. For a droplet that carried $290 \mathrm{fC}$ of net charge, there were $\sim 2 \times 10^{6}$ unpaired ions. For a droplet with net charge of $140 \mathrm{fC}$, there were $9 \times 10^{5}$ unpaired ions. This corresponds to $\sim 1.5 \mathrm{fmol}$ of extra unpaired ions added to the $290 \mathrm{fC}$ droplet. For comparison, the absolute number of moles of CHCA in a typical droplet was $\sim 300 \mathrm{fmol}$, whereas the peptides were $\sim 1-10$ amol. The identity of the ions of net excess charge could have been the conjugate acid/base pairs of CHCA, or impurity electrolytes, such as $\mathrm{Na}^{+} / \mathrm{Cl}^{-}$that existed at micromolar concentrations in solutions containing millimolar concentrations of CHCA for droplets that had positive and negative net charge respectively. These changes in the chemical composition of the levitated droplets as a result of varied $\mathrm{IP}_{\mathrm{f}}$ could be the reason for the differential quantity of precipitates formed in them. Nevertheless, we speculate that the actual identity of the ions that comprise the net charge are not critically important. Our rationale for this is that the net charge would reside at the droplet-air interface, and the possibility exists that the mechanism for the promotion of crystal nucleation and growth was heterogeneous, with those charge sites acting as "seed nuclei." This suggests that the phenomenon observed here could prove not specific to $\mathrm{CHCA}$, and nucleation of other compounds could also be promoted.

Another factor that could be important is that when the $m / z$ of the droplet is modified, so too is its mobility. Lower $\mathrm{m} / \mathrm{z}$ droplets have a higher mobility in an alternating electric field. Because the droplets are trapped at atmospheric pressure, they experience collisions passing through the air. A droplet with a higher mobility can experience more collisions and have a higher evaporation rate while levitated in an EDB [24]. Different evaporation rates between the high and low $m / z$ droplets could be the source of the promotion of crystal nucleation and growth in the highly charged droplets. Nonetheless, the observations were still dependent on the magnitude of net charge carried by the droplets, and the methodology described here is being used to study the promotion of crystal nucleation and growth in reaction vessels with varied net charge for other compounds, including other commonly employed MALDI matrices.

\section{Acknowledgments}

This research was financially supported by the Natural Sciences and Engineering Research Council (NSERC) of Canada, Waters Technologies Inc., and Simon Fraser University. MJB acknowl- 
edges an NSERC post-graduate award. The authors thank Dr. J. C. Brodovitch and Kate Scheel for their guidance in the ${ }^{32} \mathrm{P}$ experiments.

\section{References}

1. Thomson, S. W. On a self-acting apparatus for multiplying and maintaining electric charges with applications to illustrate the Voltaic theory. Proc. Royal Soc. London 1867, 16, 67-72.

2. Rayleigh, L. On the equilibrium of liquid conducting masses charged with electricity. Phil. Mag. 1882, 14, 184-186.

3. Davis, E. J.; Bridges, M. A. The Rayleigh limit of charge revisited: Light scattering from exploding droplets. J. Aerosol Sci. 1994, 25, 1179-1199.

4. Dawson, G. A. The Rayleigh instability of water drops in the presence of external electric fields. J. Geophys. Res. 1970, 75, 701-705.

5. Duft, D.; Achtzehn, T.; Muller, R.; Huber, B. A.; Leisner, T. Rayleigh jets from levitated microdroplets. Nature 2003, 421, 128.

6. Taflin, D. C.; Ward, T. L.; Davis, E. J. Electrified droplet fission and the Rayleigh limit. Langmuir 1989, 5, 376-384.

7. Widman, J. F.; Aardahl, C. L.; Davis, E. J. Observations of non-Rayleigh limit explosions of electrodynamically levitated microdroplets. Aerosol Sci. Technol. 1997, 27, 636-648.

8. Myland, J. C.; Oldham, K. B. Overcoming electroneutrality: Concentrative and electrical conditions inside a charged droplet of electrolyte solution. J. Electroanal. Chem. 2002, 522, $115-123$

9. Haddrell, A. E.; Agnes, G. R. Organic cation distributions in the residues of levitated droplets with net charge: Is the partition theory for droplets produced by an electrospray valid? Anal. Chem. 2004, 76, 53-61.

10. Wang, H. J.; Agnes, G. R. Kinetically labile equilibrium shifts induced by the electrospray process. Anal. Chem. 1999, 71, $4166-4172$.

11. Julian, R. R.; Hodyss, R.; Kinnear, B.; Jarrold, M. F.; Beauchamp, J. L. Nanocrystalline aggregation of serine detected by electrospray ionization mass spectrometry: Origin of the stable homochiral gas-phase serine octamer. J. Phys. Chem. B 2002, 106, 1219-1228.

12. Zhang, D.; Wu, L.; Koch, K. J.; Cooks, R. G. Arginine clusters generated by electrospray ionization and identified by tandem mass spectrometry. Eur. Mass Spectrom. 1999, 5, 353-361.

13. Zhang, D.; Cooks, R. G. Doubly charged cluster ions [(Na$\left.\mathrm{Cl})_{\mathrm{m}}(\mathrm{Na})_{2}\right]^{2+}$ : Magic numbers, dissociation, and structure. Int. J. Mass Spectrom. 2000, 195/196, 667-684.

14. Schalley, C. A.; Weis, P. Unusually stable magic number clusters of serine with a surprising preference for homochirality. Int. J. Mass Spectrom. 2002, 221, 9-19.
15. Vehring, R.; Aardahl, C. L.; Davis, E. J.; Schweiger, G.; Covert, D. S. Electrodynamic trapping and manipulation of particle clouds. Rev. Sci. Instrum. 1997, 68, 70-78.

16. Davis, E. J. The history of single particle levitation. Aerosol Sci. Tech. 1997, 26, 212-254.

17. Davis, E. J.; Buehler, M. F.; Ward, T. L. The double-ring electrodynamic balance for microparticle characterization. Rev. Sci. Instrum. 1990, 61, 1281-1288.

18. Gluckman, M.; Pfenninger, A.; Kruger, R.; Thierolf, M.; Karas, M.; Horneffer, V.; Hillenkamp, F.; Strupat, K. Mechanisms in MALDI analysis: Surface interaction or incorporation of analytes. Int. J. Mass Spectrom. 2001, 210/211, 121-132.

19. Bogan, M. J.; Agnes, G. R. Wall-less sample preparation of $\mu \mathrm{m}$-sized sample spots for femtomole detection limits of proteins from liquid based UV-MALDI matrices. J. Am. Soc. Mass Spectrom. 2004, 15, 486-495.

20. Bogan, M. J.; Agnes, G. R. MALDI-TOF-MS analysis of droplets prepared in an electrodynamic balance: "Wall-less" sample preparation. Anal. Chem. 2002, 74, 489-496.

21. Bogan, M. J.; Agnes, G. R. Preliminary investigation of electrodynamic charged droplet processing to couple capillary liquid chromatography with matrix-assisted laser desorption/ ionization mass spectrometry. Rapid Commun. Mass Spectrom. 2004, 18, 2673-2681.

22. Aardahl, C. L.; Vehring, R.; Davis, E. J.; Schweiger, G.; Swanson, B. D. Trapping two-particle arrays in a double ring electrodynamic balance. J. Aerosol Sci. 1997, 28, 1491-1505.

23. Paul, W. Electromagnetic traps for charged and neutral particles. Rev. Mod. Phys. 1990, 62, 531-540.

24. Zhu, J.; Zheng, F.; Laucks, M. L.; Davis, E. J. Mass transfer from oscillating spheres. J. Colloid Int. Sci. 2002, 249, 351-358.

25. Gosh, P. K.; Chattopadhyay, A. P. Ion stability and many particle interactions in a QUISTOR. Int. J. Mass Spectrom. Ion Phys. 1983, 46, 75.

26. Vedel, F.; Andre, J. Computation for ions stored in a quadrupole RF trap cooled by a buffer gas-Influence of working conditions on space-charge effects. Int. J. Mass Spectrom. Ion Processes 1985, 65, 1.

27. Guan, S. H.; Marshall, A. G. Equilibrium space charge distribution in a quadrupole ion trap. J. Am. Soc. Mass Spectrom. 1994, 5, 64-71.

28. Horneffer, V.; Forsmann, Andre; Strupat, K.; Hillenkamp, F.; Kubitscheck, U. Localization of analyte molecules in MALDI preparations by confocal laser scanning microscopy. Anal. Chem. 2001, 73, 1016-1022.

29. Sze, E. T. P.; Chan, T.-W. D.; Wang, G. Formulation of matrix solutions for use in matrix-assisted laser desorption/ionization of biomolecules. J. Am. Soc. Mass Spectrom. 1997, 9, 166-174. 